\title{
An Overview of the Marine Systems Simulator (MSS): A Simulink $^{\circledR}$ Toolbox for Marine Control Systems $\mathbb{I}$
}

\author{
T. PEREZ $* \dagger, \varnothing$. N. SMOGELI $\dagger+$, T. I. FOSSEN $\dagger \S$ and \\ A. J. SØRENSEN††
}

Keywords: Marine system dynamics, models, simulations

\begin{abstract}
The Marine Systems Simulator (MSS) is an environment which provides the necessary resources for rapid implementation of mathematical models of marine systems with focus on control system design. The simulator targets models - and provides examples ready to simulate - of different floating structures and its systems performing various operations. The platform adopted for the development of MSS is Matlab/Simulink ${ }^{\circledR}$. This allows a modular simulator structure, and the possibility of distributed development. Openness and modularity of software components have been the prioritized design principles, which enables a systematic reuse of knowledge and results in efficient tools for research and education. This paper provides an overview of the structure of the MSS, its features, current accessability, and plans for future development.
\end{abstract}

\section{Introduction}

The Marine Systems Simulator (MSS) is a Simulink ${ }^{\circledR}$-based environment that provides the necessary resources for rapid implementation of mathematical models of marine systems with focus on control system design. Its development started at the Norwegian university of Science and Technology (NTNU) and is ongoing with the help of other groups.

Since the early 1990s, significant resources have been allocated to the area of marine control systems at NTNU. This has resulted in numerous models and simulation tools developed and used by students and researchers. MSS provides an integration of these separated efforts and allows researchers and students outside NTNU to use it and contribute to further developments. The name MSS was decided in 2004 to describe the merge of three toolboxes oriented to marine system simulation: Marine GNC Toolboox, MCSim, and DCMV.

The Marine GNC toolbox was originally developed by T. I. Fossen and his students as a supporting tool for his courses on guidance and control of marine vehicles and his book (Fossen, 2002). This toolbox has provided the core library for MSS. The MCSim was a complete simulator mostly oriented toward dynamic positioning (DP) marine operations. This toolbox was initiated by A. J. Sørensen and developed by $\emptyset$. N. Smogeli and MSc students at the Department of Marine Technology at NTNU, as a source of common knowledge for new MSc and PhD students, see Sørensen et al. (2003). MCSim

*Corresponding author; Email: tristan.perez@ntnu.no

$\dagger$ Centre for Ships and Ocean Structures-CeSOS, Norwegian University of Science and Technology-NTNU, NO-7491 Trondheim, Norway.

$\ddagger$ Department of Marine Technology, Norwegian University of Science and Technology-NTNU, NO-7491 Trondheim, Norway.

§Department of Engineering Cybernetics, Norwegian University of Science and TechnologyNTNU, NO-7491 Trondheim, Norway.

IIAn early version of this paper was presented at SIMS 2005, the 46th Conference on Simulation and Modeling, Trondheim, Norway, October 13-14, 2005. 


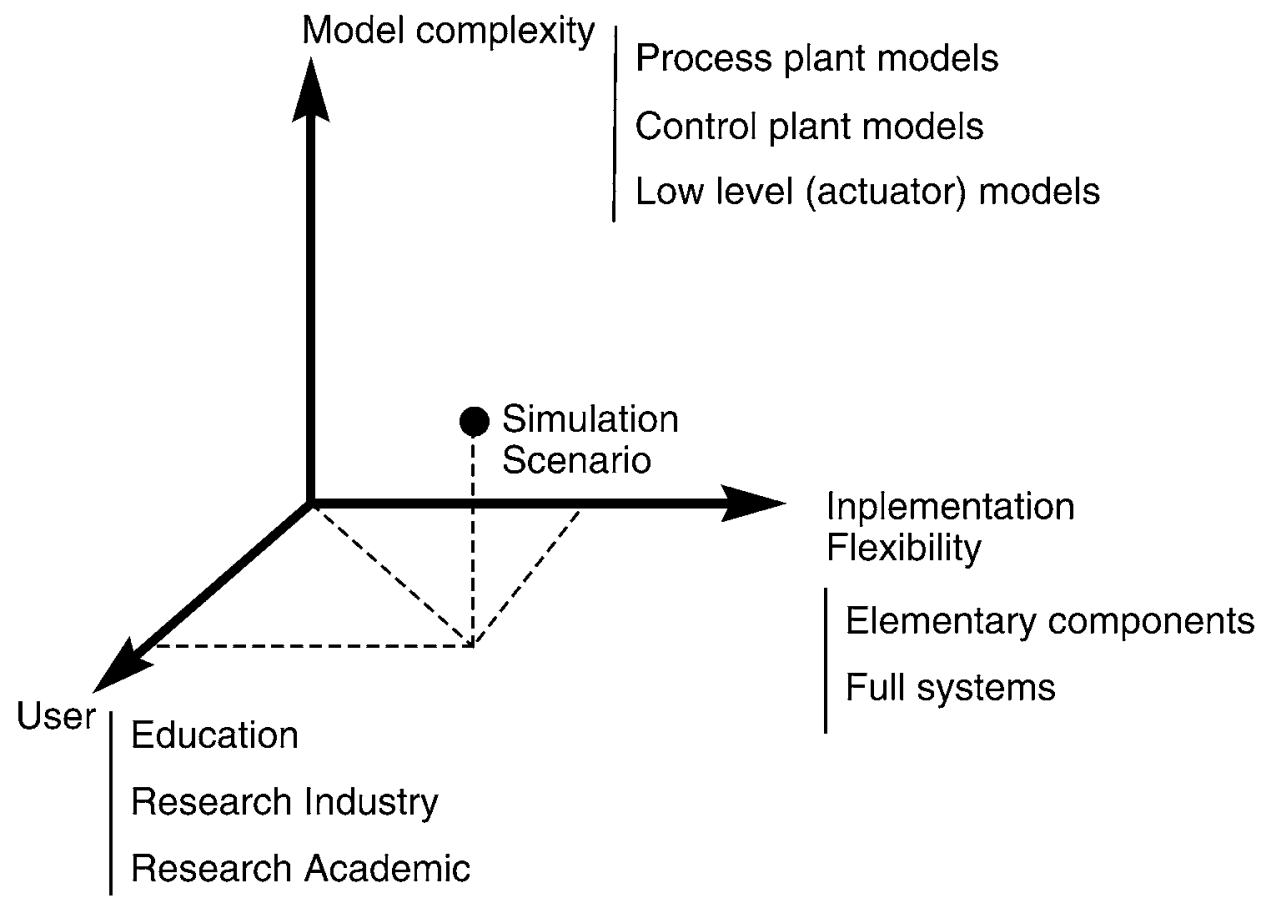

Figure 1. Simulation scenario space

incorporated sophisticated models at all levels (plant and actuator), which have now been refactored into two libraries of MSS. The DCMV toolbox was developed by Perez \& Blanke (2003) and was a reduced set of Simulink blocks oriented to autopilot design. MSS incorporates a small set of blocks from DCMV and functions related to wave spectra, and actuators.

Since the merge leading to MSS, there has been a continuous effort at NTNU by the authors and others to provide a user friendly environment which can target different simulation scenarios with the required level of complexity for different marine operations-some goals have been successfully achieved, but the work is ongoing. The rest of the paper describes the current structure of the MSS, its features, accessability, and plans for future development.

\section{MSS scope}

The scope of a simulator is defined in terms of the different simulation scenarios it can handle. These different scenarios can be further specified according to the following elements:

- Model complexity,

- Implementation flexibility,

- User proficiency.

Figure 1 illustrates this concept by making an analogy to our familiar three-dimensional space.

The model complexity is determined by the purpose of the model and its simulation. In mathematical modeling, there is a fundamental trade-off between models of great complexity, which yield a highly accurate description of many aspects of real systems 
and simplified models, which capture the fundamental aspects of the system and are mathematical tractable for analysis (Naylor \& Sell, 1982). Within the control literature complex models (process plant models) are used to test control strategies and benchmark the more simple models (control plant models) used for the design of control strategies and analysis (e.g., robustness, stability, fundamental limitations) (Goodwin et al., 2001; Sørensen, 2005b; Sørensen, 2005a; Perez, 2005).

A simulator for marine systems (ships, offshore structured, underwater vehicles, fisheries, etc.) needs to account further for the variability of the models according to the particular operation performed by the vessel, state of the sea, and speed. Changes in these system attributes result in changes in the response of the marine system, response of actuators, and control objectives, which in turn may lead to the need of different types of models. For example, consider the case of dynamic positioning (DP) of an offshore vessel. In calm to medium seas, the main control objective of the DP system could be to regulate the position (North, East and heading) by rejecting slowly varying disturbances due to mean wave forces, current and wind. As the severity of the sea state increases, the oscillatory motion induced by the waves often needs to be included as a disturbance and the reduction of pitch- and roll-induced wave oscillatory motion can be incorporated as additional control objectives. This demands changes in the models (number of DOF, and disturbance models) used by the observers and control system.

The implementation flexibility of a simulator describes whether existing models and simulation scenarios can be easily adapted to implement new types of systems using the allocated resources. This is a particularly challenging aspect of marine system simulators due to the diversity of ships and offshore structures and the operations they perform. Therefore, openness and modularity of software components have to be prioritized as design principles for systematic reuse of knowledge. In the case of MSS, Simulink ${ }^{\circledR}$ has been adopted as a development platform because of the following reasons:

- Simulink ${ }^{\circledR}$ offers the necessary modularity and ease of implementation required for a flexible simulator.

- Matlab ${ }^{\circledR}$ and Simulink ${ }^{\circledR}$ have become a tool widely used in education and research; this adds to the flexibility of MSS, because many students and scientists have access to this development platform.

- Matlab ${ }^{\circledR}$ has the great advantage that the many low-level issues have been addressed: numerical integration routines, plotting tools, data exchange and exporting facilities.

- The real-time workshop facilities of Matlab $^{\circledR} /$ Simulink $^{\circledR}$ provides a way of rapidly generating prototype controllers based on Simulink diagrams that run in real time on stand-alone computer systems for tests on scale models or full scale systems. This also offers the possibility of implementing hardware-in-the-loop simulations. This is particularly important for us, at NTNU, because students can easily implement their controllers in the scale models at the Marine Cybernetics Laboratory-see http: / /www.itk.ntnu.no/marinkyb/MCLab/

With regards to users, MSS offers different options. MSS originally evolved as a way of increasing accessibility and reuse of knowledge for students and researchers at NTNU. Different components of what now is MSS have been used in education and research for several years and it incorporates several demos of complete systems in particular operations. However, due to its modularity and organisation, MSS offers a core library of elementary blocks and functions which can be combined to generate new systems to cover research needs in both industry and academia. 


\section{Mathematical models in MSS}

To understand the software organization of MSS, let us briefly review the main components of models in a marine system. At the same time, we will introduce the main conventions adopted in MSS.

\subsection{Reference frames and magnitudes}

To describe the general motion of a marine system, we need to consider three coordinates to define translations and three coordinates to define the orientation (6DOF). These coordinates are defined using two types of reference frames: inertial frames and body-fixed frames. Figure 2 shows the different reference frames adopted in MSS, which are further specified below (Fossen, 2002; Sørensen, 2005b; Sørensen, 2005a; Perez, 2005):

- North-east-down frame ( $\boldsymbol{n}$-frame). The $n$-frame $\left(o_{n}, x_{n}, y_{n}, z_{n}\right)$ is fixed to the Earth. The positive $x_{n}$-axis points towards North, the positive $y_{n}$-axis towards East, and the positive $z_{n}$-axis towards the centre of the Earth. The origin, $o_{n}$, is located on mean water free-surface at an appropriate location. This frame is considered inertial.

- Body-fixed frame ( $b$-frame; forward-starboard-down). The $b$-frame $\left(o_{b}, x_{b}, y_{b}\right.$, $\left.z_{b}\right)$ is fixed to the hull. The positive $x_{b}$-axis points towards the bow, the positive $y_{b}$-axis points towards starboard and the positive $z_{b}$-axis points downwards. For marine vehicles, the axes of this frame are commonly chosen to coincide with the principal axes of inertia; this determines the position of the origin of the frame, $o_{b}$.

- Hydrodynamic frame ( $h$-frame; forward-starboard-down). The $h$-frame $\left(o_{h}, x_{h}\right.$, $\left.y_{h}, z_{h}\right)$ is not fixed to the hull; it moves at the average speed of the vessel following its path. The $x_{h}-y_{h}$ plane coincides with the mean-water free surface. The positive $x_{h}$-axis points forward and it is aligned with the low frequency heading angle $\psi^{1}$. The positive $y_{h}$-axis points towards starboard, and the positive $z_{h}$-axis points downwards. The origin $o_{h}$ is determined such that the $z_{h}$-axis passes through the time-average position of the centre of gravity. This frame is usually considered when the vessel travels at a constant average speed (which also includes the case of zero speed); and therefore, the wave-induced motion makes the vessel oscillate with respect to the $h$-frame. This frame is considered inertial.

Each of these frames has a specific use. For example, the $n$-frame is used to define the position and orientation of the vessel as well as the current and wind direction. The linear and angular velocity and acceleration measurements taken on board are referred to the $b$-frame, which is also used to formulate the equations of motion. The $h$-frame is used in hydrodynamics to compute the forces and motion due to the interaction between the hull and the waves in particular scenarios. These data are generally used for preliminary ship design (Couser, 2000); and therefore, it can also be used to obtain models. The $h$-frame is also used to define local wave elevation and to calculate indices related to performance of the crew or comfort of passengers-see Perez (2005).

1. The angle $\bar{\psi}$ is obtained by filtering out the 1st-order wave-induced motion (oscillatory motion), and keeping the low frequency motion, which can be either equilibrium or slowly-varying. Hence, $\bar{\psi}$ is constant for a ship sailing in a straight-line path. 


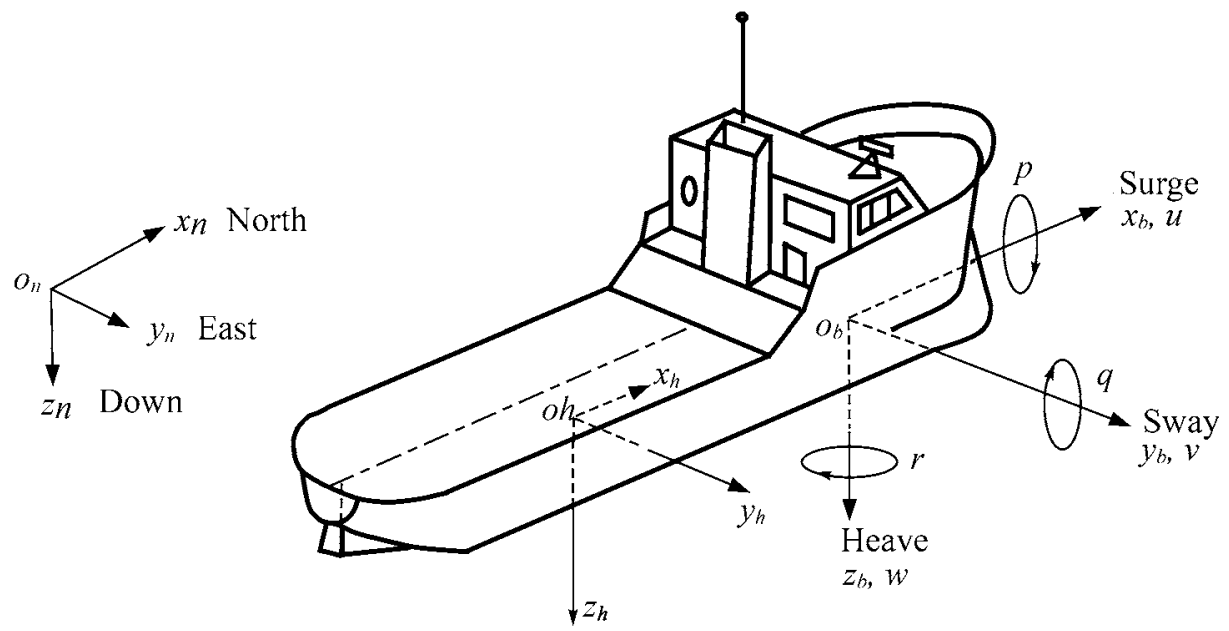

Figure 2. Notation and sign conventions for ship motion description.

The north-east-down position of a ship is defined by the coordinates of the origin of the $b$-frame, $o_{b}$, relative to the $n$-frame, expressed in the $n$-frame:

$$
\mathbf{r}_{n o_{b}}^{n} \triangleq[n, e, d]^{T} \text {. }
$$

The attitude of a ship is defined by the orientation of the $b$-frame relative to the $n$-frame. This is given by the vector of Euler angles,

$$
\Theta_{n b} \triangleq[\phi, \theta, \psi]^{T}
$$

which take the $n$-frame into the orientation of the $b$-frame. Following the notation of Fossen $(1994 ;$ 2002), the generalised position vector (or position-orientation vector) is defined as:

$$
\eta \triangleq\left[\begin{array}{l}
\mathbf{r}_{n o_{b}}^{n} \\
\Theta_{n b}
\end{array}\right]=[n, e, d, \phi, \theta, \psi]^{T}
$$

The linear and angular velocities of the ship are more conveniently expressed in the $b$-frame. The generalised velocity vector (or linear-angular velocity vector) given in the $b$-frame is defined as:

$$
v \triangleq\left[\begin{array}{c}
\mathbf{v}_{n o_{b}}^{b} \\
\omega_{n b}^{b}
\end{array}\right]=[u, v, w, p, q, r]^{T},
$$

where

- $\mathbf{v}_{n o_{b}}^{b}=[u, v, w]^{T}$ is the linear velocity of the point $o_{b}$ with respect to the $n$-frame expressed in the $b$-frame-see Figure 2.

- $\omega_{n b}^{b}=[p, q, r]^{T}$ is the angular velocity of the $b$-frame with respect to the $n$-frame expressed in the $b$-frame.

Table 1 summarizes the adopted notation. 
Table 1. Adopted nomenclature for the description of ship motion, and reference frames in which the different magnitudes are defined.

\begin{tabular}{lll}
\hline Mag. & Name & Frame \\
\hline$n$ & North position & $n$-frame \\
$e$ & East position & $n$-frame \\
$d$ & Down position & $n$-frame \\
$\phi$ & Roll ang. & Euler ang. \\
$\theta$ & Pitch ang. & Euler ang. \\
$\psi$ & Heading (yaw) ang. & Euler ang. \\
$u$ & Surge velocity & $b$-frame \\
$v$ & Sway velocity & $b$-frame \\
$w$ & Heave velocity & $b$-frame \\
$p$ & Roll rate & $b$-frame \\
$q$ & Pitch rate & $b$-frame \\
$r$ & Yaw rate & $b$-frame \\
\hline
\end{tabular}

\subsection{Equations of motion}

Using the notation of the previous section, the general form of the equations of motion of a marine system can be written in vector form as

$$
\begin{aligned}
\mathbf{M} \dot{v}+\mathbf{C}\left(v, v_{r}\right)+\mathbf{D}\left(v_{r}, \mu\right)+\mathbf{g}(\eta) & =\tau_{e n v}+\tau_{c t r l}, \\
\dot{\mu} & =A_{m} \mu+B_{m} v_{r}, \\
\dot{\eta} & =\mathbf{J}_{b}^{n}\left(\Theta_{n b}\right) v,
\end{aligned}
$$

where $M=M_{R B}^{b}+M_{A}^{b}$ is the total mass matrix (rigid body + constant added mass) with all the moments and products of inertia taken with respect to the origin of the $b$-frame. The velocity $v_{r}$ is the velocity relative to the current, i.e., $v_{r}=v-v_{c}$, where $v_{c}$ is the velocity of the current expressed in the $b$-frame. The function $\mathbf{C}\left(v, v_{r}\right)=\mathbf{C}_{R B}^{b}(v) v+\mathbf{C}_{A}^{b}\left(v_{r}\right) v_{r}$ gives the forces due to fictitious accelerations (Coriollis and Centripetal) that appear when expressing the equations of motion in a non-inertial frame. These forces have two components: one proportional to the rigid body mass and another proportional to the added mass - see Fossen (2002) for details. The terms $\mathbf{D}\left(v_{r}, \mu\right)$ are the damping terms, which can be separated into different components:

$$
\mathbf{D}\left(v_{r}, \mu\right)=D_{p 1}^{b} v_{r}+D_{p 2}^{b} \mu+D_{V i s c}^{b}\left(v_{r}\right),
$$

where the first two terms are the linear potential damping due to the energy carried away by the waves generated by the ship, and the last term accounts for viscous effects. The variables $\mu$ in the (5) account for the fluid memory effects associated with the radiation problem (waves generated by the ship). This equation together with the first two terms in (7) is a state-space representation of the convolution integral in the Cummins Equation-see Kristansen \& Egeland (2003), Fossen (2005) or Perez (2005) for further details.

The terms $\mathbf{g}(\eta)$ in (4) are the so-called restoring forces due to gravity and buoyancy, which tend to restore the up-right equilibrium position of the ship. These forces also 


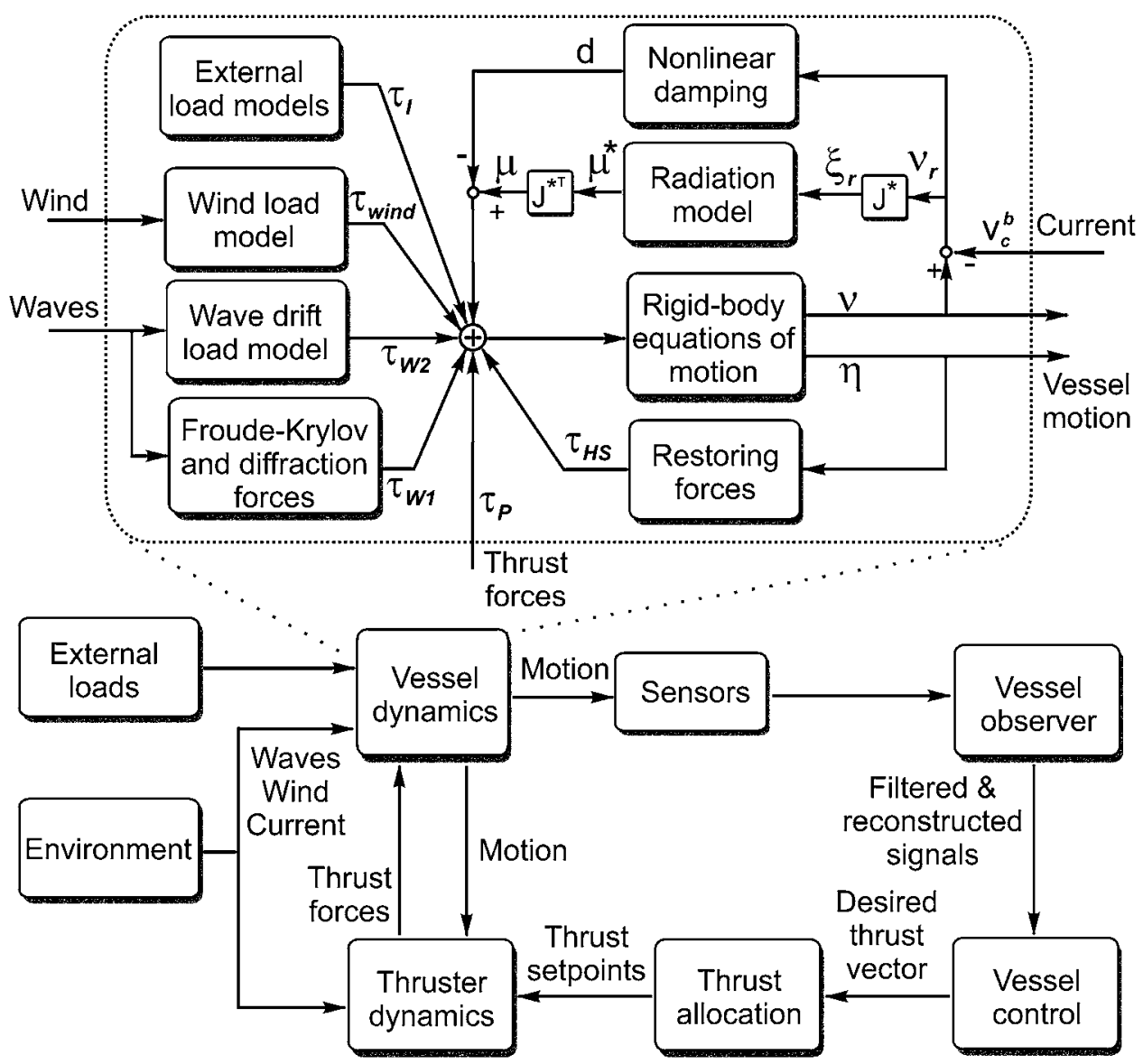

Figure 3. Marine system components

incorporate the effect of mooring systems if any. As indicated in (4), these are functions of the position and orientation of the system. Equation (6) is the kinematic transformation between the vector of linear and angular velocities in the $b$-frame and the time derivatives of the position and Euler angles - see Fossen (2002).

On the right-hand side of (4) we have the environmental excitation forces $\tau_{\text {env }}$ and the control forces $\tau_{c t r l}$. The environmental excitation forces are separated into wave, wind and current loads. The wave forces are separated into oscillatory or 1st-order wave forces (Froude-Krilov and diffraction) and the 2nd-order wave loads (mean-wave drift and slowly varying non-linear effects). The current is accounted for in the model as an offset velocity which affects the damping forces as indicated in (7) and (5).

The control forces are those generated by the different actuators: rudders, propellers, fins, thrusters, interceptors, etc. Figure 3 shows a block diagram representation of the main components of a marine system as described by equations (4)-(6)-(Smogeli et al., 2005). This figure also shows other components often included in a ship motion control system—see Fossen (2002), Sørensen (2005a) and Perez (2005) for further details about modeling of marine vehicles. 


\section{Software characteristics and organization}

MSS is organized as a set of Simulink libraries, GUIs and Matlab support functions:

- Marine GNC Toolbox,

- Add-in libraries:

- Marine Hydro

- Marine Propulsion

- Marine Systems

- Marine Visualization Toolbox (MVT),

- Matlab support functions,

The Marine $\mathrm{GNC}^{2}$ Toolbox is the core component of MSS, and most of the other components make use of it. The add-in libraries incorporate further functionality to MSS by adding more complex components. At this stage, there are three add-ins as indicated above. Some of these are still in progress, and are available upon request—see Section 5 for software availability. The Marine Visualization toolbox has recently been incorporated to MSS, but this is an independent toolbox. The integration with MSS results in additional interfaces to generate the data used by MVT.

In the following, we further describe each of the currently available components.

\subsection{Marine GNC Toolbox}

The Marine GNC Toolbox is the core component of MSS; it incorporates the simulink library marine_gnc.mdl, and its associated support functions (matlab functions). This toolbox contains the basic simulink blocks which allow implementing the models described in Section 3 and also different vessel models and examples ready to simulate.

After installing this toolbox, the entry Marine GNC Toolbox should appear in the Simulink library brouser, as shown in Figure 4.

The different blocks of the marine_gnc.mal library are organized in the following seven modules (see Figure 4):

- Control: In this module there are blocks that implement controllers for autopilots (PID controllers tuned using the Nomoto model parameters) and dynamic positioning (PID controllers for set point regulation). Future controllers incorporate hybrid controllers with motion damping capabilities (stabilizers and rudder roll stabilization)

- Environment: In this module, there are blocks to define the environmental conditions of each simulation scenario: wind, wave and current. The block waves provides all the Fourier components (amplitudes, frequencies, and wave numbers) to implement time series of a sea-surface elevation. The user can choose among four different spectra (ITTC, JONSWAP, Torsethaugen, and user specified). The block allows spreading of the spectra with respect to a mean direction and also regular wave generations. To reduce the number of Fourier components used, the block incorporates an energy-based method for eliminating components. The block also incorporates options for plotting the spectrum used in 3D, the Fourier components, and a snapshot of the sea surface generated by the chosen spectrum. 


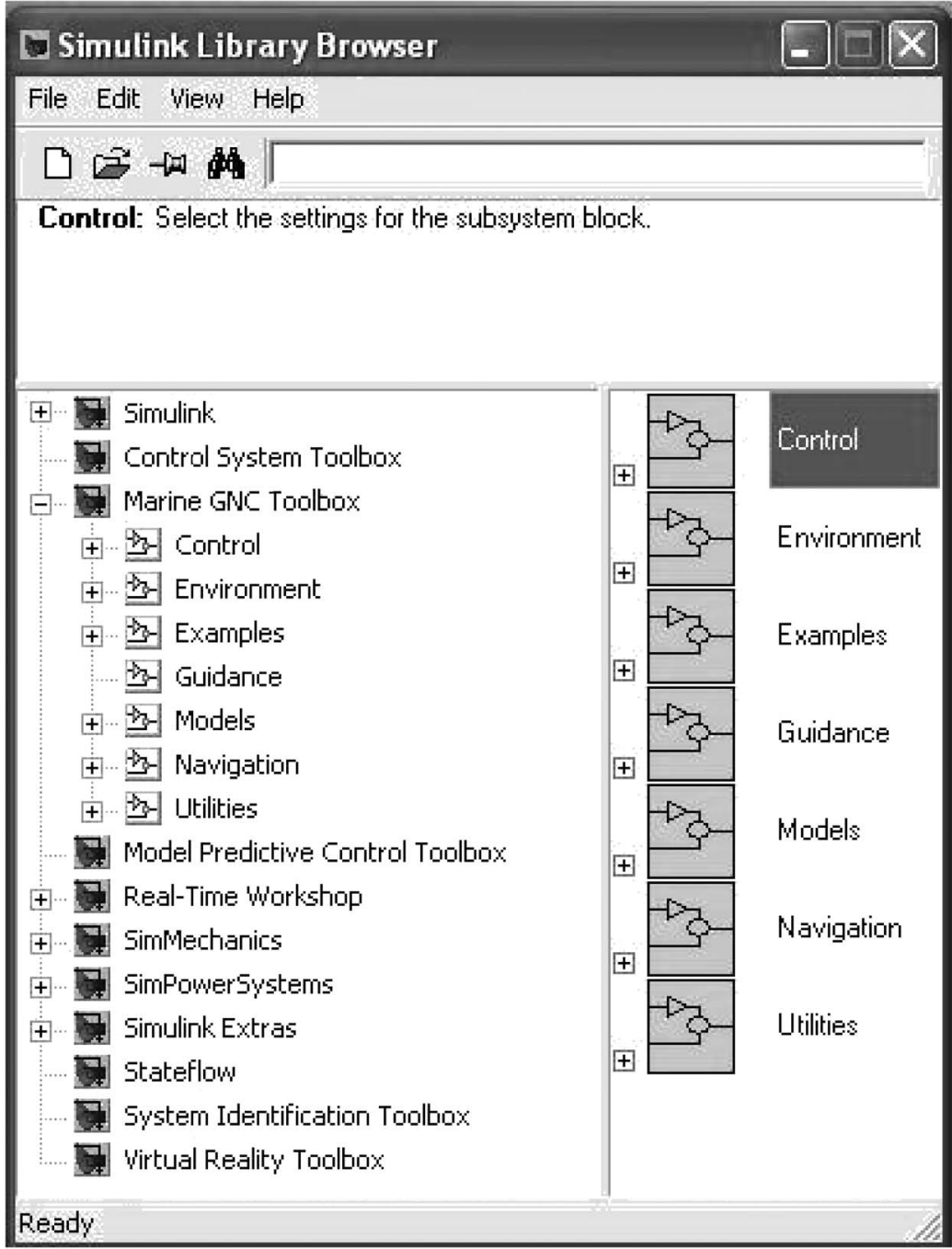

Figure 4. Simulink library browser showing the Marine GNC Toolbox after installation.

This can be used to check, before the simulations, the correct state and degree of accuracy of the sea-state description.

- Examples: In the examples module, there are seven single-block vessel models (CybershipIII, Cargo, Semisub, supply vessel, tanker, naval vessel), and ten demos ready to simulate:

-Four different autopilot designs using different measurements and wave filters. - Two DP examples.

-A thruster configuration demo for a supply vessel.

-A wave point based guidance system.

-A zig-zag test of a naval vessel.

- Turning manoeuvre of a naval vessel.

- Guidance: This module contains blocks to generate reference trajectories for DP, path following and autopilot systems. It contains reference filters, a block for 
smooth trajectory generation based on way points, and blocks to generate the rudder command for ziz-zag, pull-out, and turning tests.

- Models: This module contains all the necessary blocks to implement the equations of motion (4)-(6). This includes blocks for kinematic transformations and equations of motion in 3, 4 and 6DOF, control surfaces (rudders and fins), environmental load blocks (which combined with the environment blocks provide the loads.) This module also incorporate basic propulsion blocks with open water characteristics of propellers, propeller shaft dynamics, and propeller configuration blocks for different DP vessels.

- Navigation: This module contains passivity- and Kalman-based observers. Future version will incorporate other filters, models of different sensors (GPS, HPS, Microwave Systems, VRU, IMU, etc.)

- Utilities: This module includes blocks that perform mathematical operations to related signals.

\subsection{Marine Hydro add-in}

One of the most difficult issues for anyone attempting a control system design for a marine system is to obtain an appropriate mathematical model. The Marine Hydro add-in provides an interface with commonly used hydrodynamic codes. These codes are widely used by Naval architects during preliminary stages of ship design, and provides most of the parameters necessary to implement a model of a marine system that could be used as an initial step in the control system design process.

The Marine Hydro add-in provides matlab functions that read the output files of different hydrodynamic codes: ShipX-VERES (Fathi, 2004), SEAWAY (Jouernee \& Adegeest, 2003), and WAMIT $^{3}$ (WAMIT, 2004), and generate a predefined data structure. These codes use a geometrical definition of the hull and its loading condition (distribution of mass) to calculate the following data: restoring coefficients, added-mass and damping coefficients, exciting forces transfer functions (force-RAOs), body-motion transfer functions (motion-RAOs), local hydrodynamic pressure, mean-drift force and moment, etc. All these data are commonly exported in ASCII form in different files, and the Marine Hydro add-in provides Matlab routines that extract all this information and generate a data structure vessel for the particular ship analyzed. Once vessel is generated, there are different functions to plot data (added mass and damping as a function of the frequency and RAOs), functions to generate a state-space model for the fluid memory effects, and a a special simulink model for time domain simulations. The latter provides a seamless interface for a rapid implementation of models of marine vessels in three steps:

1. Load the ship data into a particular hydrodynamic code (at this stage VERES and SEAWAY), and execute it.

2. Import the hydrodynamic data generated by the codes into Matlab ${ }^{\circledR}$ using the functions of the add-in. This defines the structure vessel in matlab.

3. Use the Hydro add-in simulink model for time-domain simulations in different sea states.

Figure 5 illustrates the process. Examples of use of this add-in have been described in (Fossen \& Smogeli, 2004) and (Smogeli et al., 2005) 


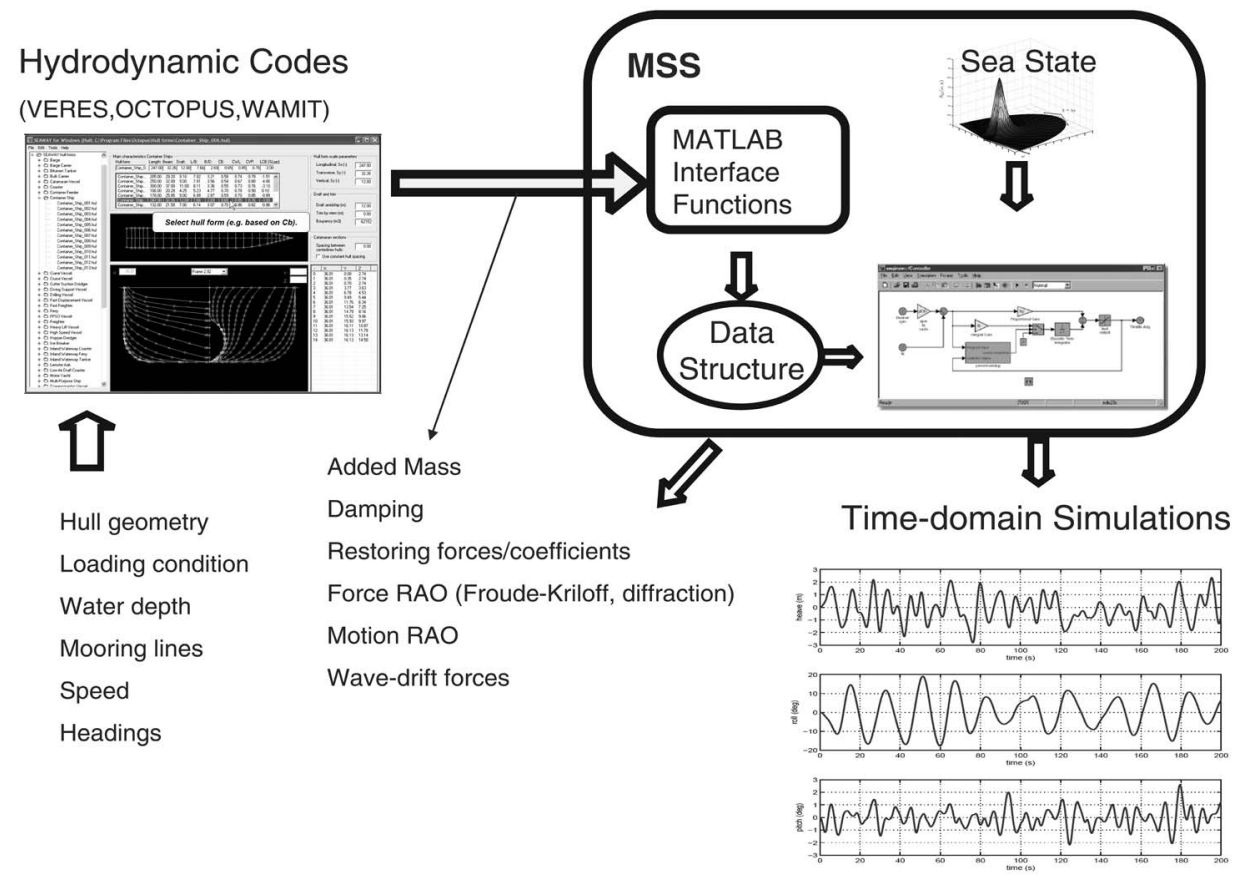

Figure 5. Marine Hydro capabilities.

NOTE: The Marine Hydro add-in DOES NOT provide licences and/or copies of the hydrodynamic codes for which the interfaces have been developed. The user should obtain appropriate licensed copies of these codes.

\subsection{Marine Propulsion add-in}

The MSS Marine Propulsion Add-In targets simulation and control design for propellers, thrusters, and rudders. The current library consists of blocks for propeller characteristics, various hydrodynamic calculations and thrust loss models, motor models, sensors, control systems, and a variety of other tools. In addition, complete models compiled from the basic blocks are included.

Five different models of the propeller hydrodynamics have been implemented, producing the nominal (open-water) propeller thrust and torque. Two model representations for open and ducted fixed-pitch propellers (FPP), one 4-quadrant and one 1-quadrant model, are available, taking propeller shaft speed and propeller advance velocity as input. Similarly, two model representations for open and ducted controllable pitch propellers (CPP), one 4-quadrant and one 1-quadrant, are available, taking propeller shaft speed, propeller advance velocity, and propeller pitch as input. The 1-quadrant model is also suitable for CPP tunnel thrusters. Finally, a cavitation number based model for FPP tunnel thrusters is available, taking propeller shaft speed and tunnel submergence as input. Note that all models require the user to input some kind of propeller characteris- 
tics, either from known propeller series, from a propeller analysis program, or from experiments.

Thrust losses due to the presence of the hull in terms of wake and thrust deduction is accounted for, as well as loss effects due to variations in advance velocity, cross-coupling drag for open and ducted propellers due to transverse fluid velocity, speed loss for tunnel thrusters due to vessel surge velocity, and ventilation and in-and-out-of-water effects, including the Wagner effect. The relative motion of the thruster with respect to the water is calculated from vessel 6 DOF motion, waves and current. Additional loss effects, e.g. the Coanda effect and thruster-thruster interaction, are under development.

Simplified models of the thruster motors, as well as azimuth and pitch dynamics are available, including rate saturation and physical limits. A propeller shaft model is also present, including friction and shaft inertia.

Blocks for conventional shaft speed control, torque control, power control, and combined power/torque control are available. Sensor models including white noise and quantizing are also available in order to give realistic feedback signals.

A thruster dynamics module, compiled of the various propeller characteristics, loss effects, and motor models has been included in the library. This shows how the basic building blocks can be used to build more complex models. A closed-loop model, including sensors and control systems, is also available. These modules require extensive user input for use, and are intended for in-house research on propulsion control.

\subsection{Marine Systems add-in}

The Marine Systems add-in is a simulink library with complex system ready to simulate-which has not yet been released. This library is to be used mostly for education purposes as an aid to illustrate concepts in class and by letting students experiment with what they have learned instead of fighting the common (and necessary) blunders they make while implementing complex systems in Simulink. This library can also be used for application of novel control and identification techniques on benchmark examples.

\subsection{Marine Visualization Toolbox (add-in)}

The Marine Visualization Toolbox MVT add-in displays data from simulations, experiments or measurements of marine systems as 3D animations. The animations may be viewed on-line or saved to file. This toolbox was developed at the Department of Engineering Cybernetics NTNU (Danielsen et al., 2004), and is available independent of MSS, but integration with MSS is ongoing.

MVT uses features of the Matlab Virtual Reality Toolbox, which is an interface for viewing 3D models. The resulting animations depict vessel moving and rotating according to the time-varying input data. Figure 6 shows a snapshot of one of such animations for scale vessel on a replenishment operation (Kyrkjebø \& Pettersen, 2003). The input data may represent a maximum of six degrees of freedom (6 DOF) in terms of the xyz-positions and Euler angles. With the current version of MVT, these data need to be assembled into a special strucutre by the user. However, work is currently being done on a special simulink block that takes the velocity and position vectors $v\left(t_{k}\right)$ and $\eta\left(t_{k}\right)$ and save the data into the special structure to be then used by MVT. Any number of vessels may be animated at the same time. The vessels are animated in a scene model (e.g. a coastline or a harbor) representing the actual surroundings of the simulations, 


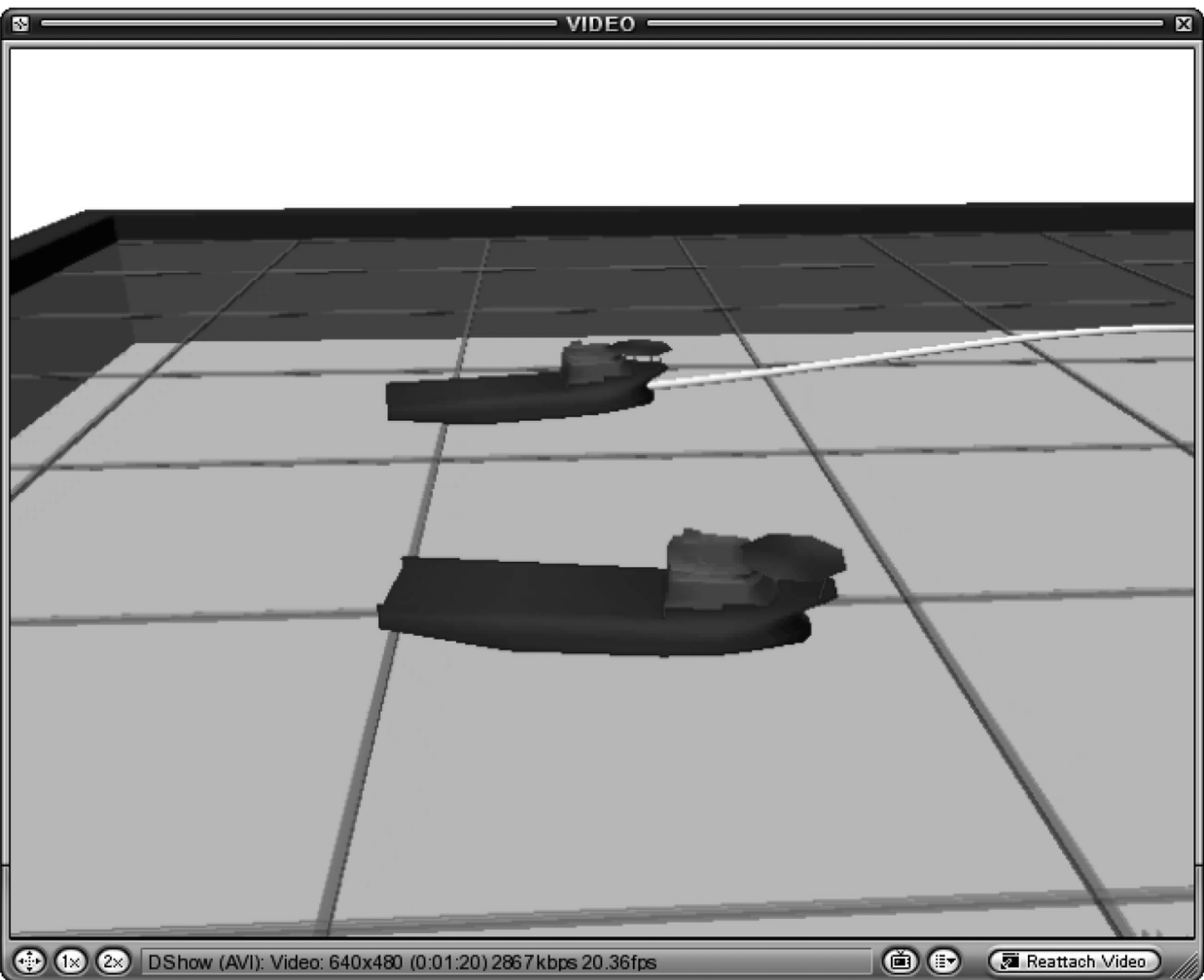

Figure 6. Snapshot of an MVT animation based on experiments of a scale vessel on a replenishment operation.

experiments or full scale measurements. Both scene and vessel models are implemented in the Virtual Reality Modeling Language (VRML).

MVT includes two libraries of VRML files; vessel models and scenes. Combinations of these are generated to resemble specific scenarios with the desired number and types of vessels located in the desired surroundings. For further details see Danielsen et al. (2004) and the links at the MSS web site (see next section).

\section{Where to find it and Access Level}

MSS is free, i.e., there is no fee for using MSS; however, some of its components may be of restricted distribution. The only conditions for the use of any of the MSS components is the acknowledgement of the publication of any result obtained using MSS, and reporting back any bugs and contributions. The current version of MSS, and conditions for use can be found at:

\section{http://www.cesos.ntnu.no/mss /}

In this web site, there is information about software characteristics, instructions for installation, previously released versions, links to related literature, and technical reports.

At this stage, there are two access levels:

- Free: This incorporates the Marine GNC Toolbox and all its support functions. 
- Free Restricted: As MSS is to be used for research, as well as for education, some models may be sensitive and kept either in-house or be shared only by collaborators. Therefore, some elements of MSS will be of restrictive distribution. The hydro and propulsion add-ins are currently restricted to collaborators, and can be made available upon request.

\section{Elementary blocks and guidelines for contributions}

Simulink blocks are elementary constitutive parts of the MSS simulink libraries, and are used to create other more complex blocks or models or both. A masked block serves to establish a boundary for the modular modeling approach. This boundary is set by specifying the following attributes of the block:

- Block name,

- Inputs and outputs name,

- Mask information,

- Help information.

The rules followed in the development of MSS are indicated next.

\subsection{Color code}

When users create a new block they should name it and mask it. Under the mask, the following color code is to be used for easy recognition of elementary blocks functions:

- Green-input port

- Red-output port

- Grey-logic

- Yellow-others

\subsection{Name and ports}

The block name is the first indication of the task performed. The Inputs and output labels indicate how to interconnect the block with other existing blocks: they share the same labels. Do not include units in the port labels, and Do not use the drop shadow option of format.

\subsection{Dialog window}

The dialog window is generated by the mask, and allows the user to enter parameters to the block. Figure 7 shows an example.

We recommend every block to be masked even when it does not require parameters; this helps modularity and more importantly it provides access to the help of the block. The following information should be incorporated in the dialog window:

- Brief Description and Formulae (if short).

- Inputs and outputs description with units in SI and positive convention.

- Parameter description (if short, if not this goes in the help).

- Copyright (C) 200X, NTNU (if you would like your contribution to be incorporated in MSS you have to give the copyright to NTNU.)

- Authors Name. 


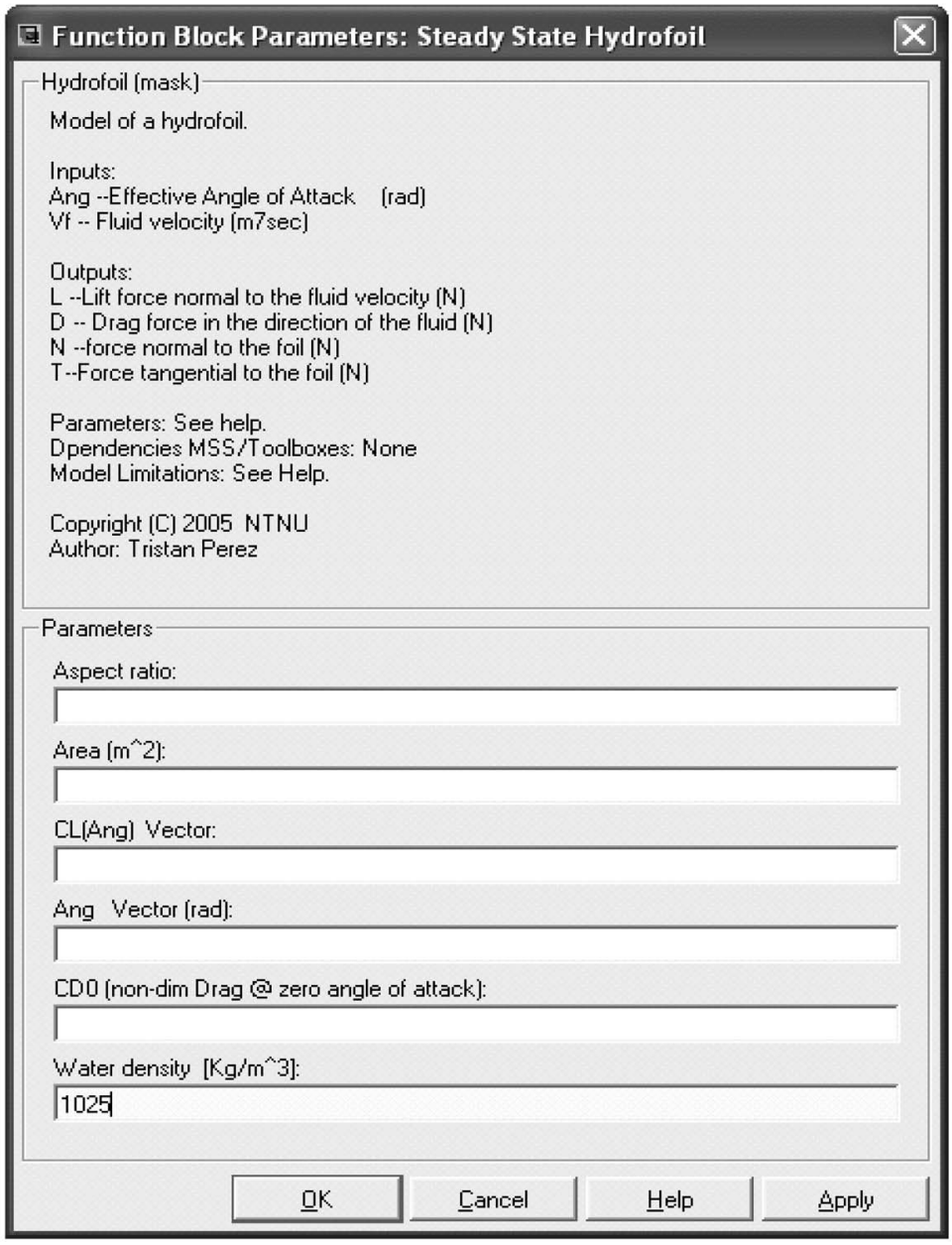

Figure 7. Example of dialog window.

NOTE: Incorporate SI units in the parameter window but not in the block port labels.

\subsection{Help window}

The help window is called from the dialog window of the block. The help provides further description of block functionality. Due to the potential of multi-user development, MSS will not incorporate a user manual, and all the help should be included in the mask.

The documentation of the block may be written in HTML and the code pasted in the help window of the mask. Alternatively, users can generate an HTML file and call it from the mask using:

web ( [ 'file: / 'which ('Help_blockName.htm' ) ] );

The help documentation should include:

- Model limitations and validity range, 
- Dependencies (If it incorporates other blocks),

- References to the literature.

\section{Summary}

In this paper, we have provided an overview of MSS. This included a description of its structure, its features, current accessability, and plans for future development. Because MSS is an ongoing project and we would like users to contribute to it, we have also described a small set of rules that we are trying to follow to have a consistent development. Check the MSS web site for further information, future developments, and the most recent release of its components: http://www. cesos.ntnu.no/mss/

\section{Acknowledgements}

The development of the material hereby presented has been supported by the Centre for Ships and Ocean Structures-CeSOS and its main sponsor: The Research Council of Norway. The authors also acknowledge the collaboration of Andreas Danielsen, Erik Kyrkjebø and Prof. Kristin Pettersen from Dept. of Eng. Cybernetics NTNU who developed the Marine Visualization Toolbox and are now working on a special version to be released with MSS.

\section{References}

Couser, P. (2000). Seakeeping analysis for preliminary design. Ausmarine 2000, Fremantle W.A., Baird Publications.

Danielsen, A. L., Kyrkjebø, E. \& Pettersen, K. Y. (2004). MVT: A marine visualization toolbox for MATLAB”. In: IFAC Conference on Control Applications in Marine System, Ancona, Italy.

FATHI, D. (2004). ShipX Vessel Responses (VERES). Marintek AS Trondheim. http:// www.marintek.sintef.no/.

Fossen, T. I. (1994). Guidance and Control of Ocean Marine Vehicles. John Wiley and Sons Ltd. New York.

Fossen, T. I. (2002). Marine Control Systems: Guidance, Navigation and Control of Ships, Rigs and Underwater Vehicles. Marine Cybernetics, Trondheim.

Fossen, T. I. (2005). A nonlinear unified state-space model for ship maneuvreing and control in a seaway. In: International Journal of Bifurcation and Chaos. Vol. 15. pp. 2717-2746.

Fossen, T. I. \& SMOgeli, Ø. N. (2004). Nonlinear time-domain strip theory formulation for low speed manoeuvring and station-keeping. Modelling Identification and Control-MIC.

Goodwin, G. C., Graebe, S. \& Salgado, M. (2001). Control System Design. Prentice-Hall, Inc.

Jouernee, J. M. J. \& Adegeest, L. J. M. (2003). Theoretical Manual of Strip Theory Program SEAWAY for Windows. TU Delft, Delft University of Technology. www.ocp.tudelft.nl/mt/ journee.

Kristansen, E. \& Egeland, O. (2003). Frequency dependent added mass in models for controller design for wave motion ship damping. In: 6th IFAC Conference on Manoeuvring and Control of Marine Craft MCMC'03, Girona, Spain.

KyrkJebø, E. \& Pettersen, K. Y. (2003). Ship replenishment using synchronization control. In: 5th IFAC Conference on Manoeuvring and Control of Marine Craft MCMC'03.

NAYloR, A.W. \& Sell, G. R. (1982). Linear Operator Theory in Engineering and Science. Vol. 40 of Applied Mathematical Sciences. Springer-Verlag.

Perez, T. (2005). Ship Motion Cotnrol: Course Keeping and Roll Reduction using rudder and fins. Advances in Industrial Control. Springer-Verlag, London.

Perez, T. \& BlanKe, M. (2003). DCMV a matlab/simulink toolbox for dynamics and control of marine vehicles. In: 6th IFAC Conference on Manoeuvring and Control of Marine Craft $M C M C^{\prime} 03$. 
Smogeli, Ø. N., Perez, T., Fossen, T. I. \& Sørensen, A. J. (2005). The marine systems simulator state-space model representation for dynamically positioned surface vessels. In: International Maritime Association of the Mediterranean IMAM Conference, Lisbon, Portugal.

Sørensen, A. J. (2005a). Marine cybernetics, modelling and control. Lecture notes UK-05-76. Department of Marine Technology, NTNU, Trondheim, Norway.

Sørensen, A. J. (2005b). Structural properties in the design and operation of marine control systems. IFAC Journal on Annual Reviews in Control, Elsevier Ltd. 29(1), 125-149.

Sørensen, A. J., Pedersen, E. \& Smogeli, Ø. N. (2003). Simulation-based design and testing of dynamically positioned marine vessels. In: International Conference on Marine Simulation and Ship Maneuverability (MARSIM), Japan.

WAMIT (2004). WAMIT User Manual. www.wamit.com. 